\title{
E. coli claims the driving seat for cancer
}

Cf

colibactin is a requirement for

progression to cancer.
In recent years, mounting evidence has implicated the microbiota in various inflammatory diseases, but whether these commensal bacteria have an active role in the development of cancer has been unclear. Now, Arthur et al. provide evidence that inflammation alters the intestinal microbiota by favouring the proliferation of genotoxic commensals, and that the Escherichia coli genotoxin colibactin promotes colorectal cancer (CRC).

To learn more about the link between the microbiota, inflammation and carcinogenesis, the authors began by comparing the intestinal microbial composition of interleukin-10-deficient $\left(/ 110^{-/-}\right)$mice (which are susceptible to colitis) with that of wild-type mice, in the presence and absence of the

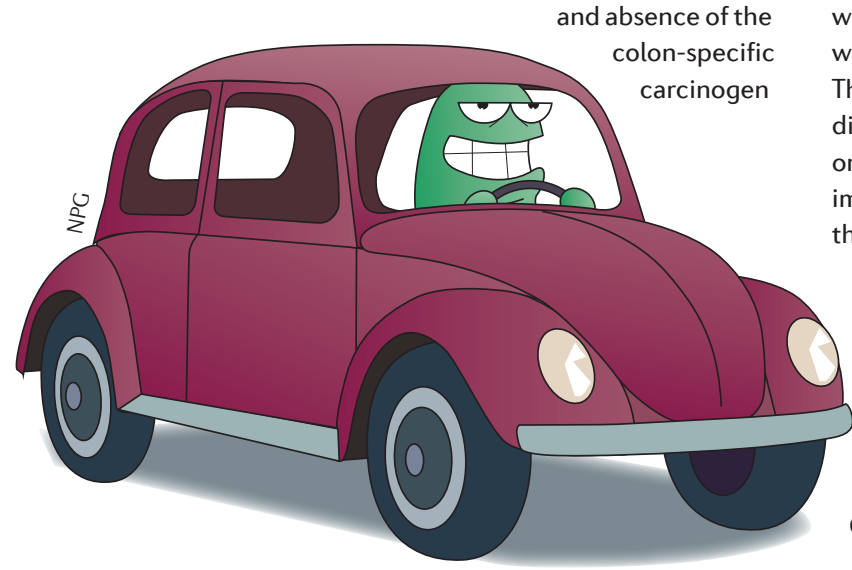

azoxymethane (AOM). They found that in $1110^{-/-}$mice the microbiota was less diverse but that the prevalence of commensal E. coli was 100 -fold higher than in wild-type mice. Importantly, the observed shift in microbial composition was independent of AOM treatment, indicating that inflammation (and not cancer) was the cause of the modified microbiota in $/ l 10^{-/-}$mice.

To examine the effect of $E$. coli on CRC development, AOM-treated germ-free $I l 10^{-/-}$mice were colonized with either $E$. coli str. NC101 (a mouse commensal strain) or Enterococcus faecalis (a human commensal species). Histological analysis revealed that, despite both mouse groups having similar levels of colitis, E. faecalis-colonized mice rarely showed signs of tumorigenesis, whereas $80 \%$ of the mice colonized with $E$. coli developed invasive tumours. This effect was independent of any differences in the levels of cytokines or infiltrating immunological cells, implicating an E. coli-specific factor as the tumour-promoting agent.

The polyketide synthase ( $p k s$ ) pathogenicity island, encoding the DNA-damaging toxin colibactin, was detected in the genome of $E$. coli str. NC101 and was also found in almost $70 \%$ of intestinal $E$. coli strains from human patients with CRC, making this island a potential candidate for the tumour-promoting agent. To test this hypothesis in vivo, the authors examined the ability of an E. coli str. NC101 mutant lacking pks (NC101 $\Delta$ pks) to cause tumorigenesis in $\mathrm{AOM}$-treated $/ 110^{-/}$mice. Like the wild-type strain, NC101 $\Delta$ pks induced chronic inflammation, but for the mutant this occurred in the absence of invasive tumours, indicating that colibactin is a requirement for progression to cancer. In addition, the NC101 $\Delta$ pks mutant induced less DNA damage in mouse colonocytes than in the wild-type strain.

Together, these data demonstrate the complex interplay between inflammation and the microbiota in driving CRC, and provide further support for the 'pathobiont' model, which posits that chronic inflammatory disease triggers the pathogenic potential of resident commensals. This study suggests that inflammation-induced changes in the gut microbiota can have a direct influence on the development of CRC. It also identifies pks-containing $E$. coli as a new risk factor for CRC, although the authors note that additional studies using other models will be needed to confirm this link.

Christina Tobin Kåhrström Associate Editor, Nature Reviews Microbiology

This article originally appeared in Nature Rev. Microbiol. (doi:10.1038/nrm2878).

ORIGINAL RESEARCH PAPER Arthur, J. C. et al. Intestinal inflammation targets cancer-inducing activity of the microbiota. Science 16 Aug 2012 (doi:10.1126/science.1224820)

FURTHER READING Chow, J. \& Mazmanian, S. K. A pathobiont of the microbiota balances host colonization and intestinal inflammation. Cell Host Microbe 7, 265-276 (2010) 